\title{
The expanding pattern of Aedes aegypti in southern Yunnan, China: insights from microsatellite and mitochondrial DNA markers
}

\author{
Pengbo Liu' ${ }^{1}$ Liang Lư ${ }^{1 *}$, Jinyong Jiang ${ }^{2}$, Yuhong Guo ${ }^{1}$, Mingdong Yang ${ }^{2}$ and Qiyong Liu ${ }^{1 *}$
}

\begin{abstract}
Background: Aedes aegypti, the vector of dengue fever, was first reported in Yunnan in 2002. Now, this species is found in nine counties in border areas of south-west Yunnan. Related dengue fever outbreaks have been reported since 2013. The population genetics of Ae. aegypti in these areas were studied to explain the expansion history of this species.

Methods: Fifteen natural populations of Ae. aegypti were sampled from six counties of Yunnan, and two laboratory populations from Guangdong and Hainan were also included in this study. A total of 12 microsatellite loci and three mitochondrial genes were analysed.

Results: The results indicate that Ae. aegypti populations from Yunnan show similar genetic diversity. The 17 populations could be divided into three groups: the first group included populations from Longchuan, Ruili and Gengma, which are located in the southwest of Yunnan; the second group included populations from Jinghong and Menghai, in the south of Yunnan; and the third group included populations from Mengla and the two laboratory populations from Guangdong and Hainan. Both microsatellite and mtDNA data revealed that the genetic relationships of the populations corresponded to their geographic relationships.

Conclusions: The results suggested that the expansion of Ae. aegypti from northern Myanmar and Laos to southern and southwestern Yunnan was a natural process. The effect of human activity on expansion was not obvious. Surveillance efforts should still be focused on border areas where Ae. aegypti does not occur, and a powerful control strategy should be applied to prevent outbreaks of dengue fever.
\end{abstract}

Keywords: Aedes aegypti, Population genetics, Microsatellite loci, Expansion, Southwestern China

\section{Background}

Dengue fever (DF) is the most rapidly spreading arboviral disease in the world, with Aedes aegypti representing the most important vector [1]. In 2009, the World Health Organization (WHO) reported that there were approximately 50 million new cases of dengue per year, and approximately 2.5 billion people are at risk of contracting

\footnotetext{
*Correspondence: luliang@icdc.cn; liuqiyong@icdc.cn

1 State Key Laboratory of Infectious Disease Prevention and Control, National Institute for Communicable Disease Control and Prevention, Chinese Center for Disease Control and Prevention, Beijing 102206, China Full list of author information is available at the end of the article
}

DF around the world [2]. Bhatt et al. [3] estimated in 2013 that there were approximately 390 million dengue infections per year, and approximately $40 \%$ of the world population is at risk of infection from dengue. The latest WHO information indicated that the incidence of DF had increased 30 -fold over the last 50 years and up to 50-100 million infections were estimated to occur annually in over 100 endemic countries, putting almost half of the world's population at risk [4].

Before 2000, the distribution of Ae. aegypti in China was recorded in areas below $22^{\circ}$ north latitude, including Hainan, Guangxi, Guangdong and Taiwan, and no 
Ae. aegypti were recorded in Yunnan [5]. This species was first found at the Jiegao Port of Ruili, a county south-west of Yunnan, in 2002 [6]. In 2006, Ae. aegypti was found in the county city of Ruili, which is approximately $5 \mathrm{~km}$ away from Jiegao Port. In 2008, and 2009, Ae. aegypti was found at the Guanlei wharf on the Mekong River and in the Mohan Port of Mengla County, respectively. In 2011, Ae. aegypti was found in Jinghong, Xishuangbanna. There were records of Ae. aegypti in Ruili, Mangshi, Mengla, Menghai, Jinghong, Yingjiang, Longchuan and Gengma counties by 2014 [7]. Dengue outbreaks have since been reported almost every year in Ruili, Jinghong, Mengla and Gengma in Yunnan $[8,9]$.

Yunnan is located in southwestern China in areas contiguous with Myanmar, Laos, and Vietnam and connected to Cambodia and Thailand via the Mekong River. These countries of Southeast Asia are all threatened by dengue fever transmitted by Ae. aegypti [10]. Most imported dengue fever cases in China arise from infections contracted in Southeast Asia [11]. Human travel and trade between Southeast Asia and China might not only export dengue fever cases to China but also export Ae. aegypti populations to border areas of Yunnan. However, the real reasons for the appearance of Ae. aegypti populations in Yunnan should be examined to develop an effective survey and control strategy for Ae. aegypti in related areas. There are two possible scenarios for the invasion of Ae. aegypti in Yunnan, China: (i) the mosquito expanded to Yunnan naturally, and populations from different areas are genetically independent; and (ii) the mosquito invaded Yunnan due to increasing transportation among countries, and populations from different areas are therefore linked by the highway system between countries and counties of Yunnan.

Because of the high variability, codominant expression, and broad genome distribution of microsatellites, they have been widely used as classic genetic markers in population genetic studies, providing information on population structure, patterns of gene flow, histories of introduction and colonization, or pathways of the expansion of invading species in new areas [12,13]. There have been several reports of the successful development and use of microsatellite markers in Ae. aegypti from South America and Southeast Asia and on a global scale [1417]. However, almost half of the microsatellite sequences of Ae. aegypti are located directly within or closely linked to repetitive elements, which could reduce the possibility of using these microsatellite sequences in genetic studies [16]. Therefore, in the present study, we isolated new microsatellites and validated their application in genetic research in Ae aegypti populations of China.

Mitochondrial DNA (mtDNA) replicates independently from nuclear genomes and is materially inherited without recombination, so it can allow sharper genetic differentiation than nuclear DNA $[18,19]$. The mitochondrial genes cytochrome $c$ oxidase subunit 1 ( $\operatorname{cox} 1)$, $\mathrm{NADH}$ dehydrogenase subunit 4 (nad4), and NADH dehydrogenase subunit 5 (nad5) are frequently used to infer the phylogenetic relationships of mosquitoes. cox 1 is the most conserved of the three cytochrome oxidases encoded by mtDNA [20] and shows greater sequence variation at the inter-species level than at the intra-species level [21]. The nad4 and nad5 loci are highly polymorphic and have often been used to conduct genetic analysis of Ae. aegypti [22-24].

Based on population genetics analysis with microsatellite and mtDNA loci, we sought to study two issues in the Ae. aegypti population in Yunnan, China: (i) the scenario of Ae. aegypti invasion of Yunnan, to develop an effective strategy for the surveillance and control of the Ae. aegypti population; and (ii) considering the expansion of Ae. aegypti, the possibility that this species will continue to spread within and outside of Yunnan. Molecular markers that can be used for population identification are needed to distinguish Ae. aegypti populations from Yunnan, Guangdong and Hainan.

\section{Methods \\ Mosquito samples}

Mosquitoes from fifteen sites in Yunnan were sampled in the autumn of 2016 based on the distribution of $A e$. aegypti: four from Ruili, one from Longchuan, two from Mengla, one from Menghai, one from Gengma, and six from Jinghong (Fig. 1, Table 1). Adult mosquitoes were collected using a sweep net from each sampling area, and the larvae and pupae were collected with ovitraps. Each sampling site was located with GPS. Samples of 2 laboratory populations from Guangdong and Hainan, which were collected from the distribution areas in the two provinces, were also used in this research (Fig. 1, Table 1). Samples identified as Ae. aegypti were preserved in $95 \%$ ethanol in labelled vials and stored at $4{ }^{\circ} \mathrm{C}$ before the isolation of genomic DNA.

\section{DNA extraction}

Total nucleic acids were extracted from each individual of Ae. aegypti with a magnetic bead-based semi-automatic system (Biotake, Beijing, China) following the manufacturer's protocol. The DNA samples were stored at $-20^{\circ} \mathrm{C}$ until further analysis.

\section{Microsatellite analysis}

Twelve polymorphic microsatellite loci were used in this study: 11 pairs of primers were obtained via a genomebased method [16], and 1 pair of primers was selected from reported data [25]. The primer sequences and 




1

$\begin{array}{llllll}0 & 20 & 40 & 80 & 120 & 160\end{array}$
Legend

- Sample areas

Dengue fever positive areas

Fig. 1 Sampling locations in Yunan ( $n=15)$, Guangdong $(n=1)$ and Hainan $(n=1)$. Specifically, in Yunan, there was 1 sampling site in Longchuan, 4 in Ruili, 1 in Gengma, 1 in Menghai, 6 in Jinghong and 2 in Mengla county. In 2016, Ae. aegypti was reported from these counties. Among these counties, Ruili, Gengma, Jinghong and Mengla were dengue fever-positive areas and are labelled in yellow 
Table 1 Sampling information for the Ae. aegypti populations used in the present study

\begin{tabular}{llll}
\hline $\begin{array}{l}\text { Population code } \\
\text { collection site) }\end{array}$ & Region & $n$ & Geographical coordinates \\
\hline MML (Mengmao Road) & Ruili & 47 & $24^{\circ} 00^{\prime} 57^{\prime \prime} \mathrm{N}, 97^{\circ} 50^{\prime} 39^{\prime \prime} \mathrm{E}$ \\
NAM/Nanmen & Ruili & 35 & $24^{\circ} 01^{\prime} 52^{\prime \prime} \mathrm{N}, 97^{\circ} 48^{\prime} 18^{\prime \prime} \mathrm{E}$ \\
RJL/Ruijin Road & Ruili & 32 & $24^{\circ} 01^{\prime} 09^{\prime \prime} \mathrm{N}, 97^{\circ} 51^{\prime} 27^{\prime \prime} \mathrm{E}$ \\
JGL/Jiegang Road & Ruili & 47 & $24^{\circ} 00^{\prime} 59^{\prime \prime} \mathrm{N}, 97^{\circ} 51^{\prime} 35^{\prime \prime} \mathrm{E}$ \\
LYC/Laying village & Longchuan & 32 & $24^{\circ} 11^{\prime} 50^{\prime \prime} \mathrm{N}, 97^{\circ} 43^{\prime} 38^{\prime \prime} \mathrm{E}$ \\
GEM/Mengding & Gengma & 24 & $22^{\circ} 00^{\prime} 21^{\prime \prime} \mathrm{N}, 99^{\circ} 04^{\prime} 58^{\prime \prime} \mathrm{E}$ \\
LBH/Department store & Mengla & 28 & $21^{\circ} 27^{\prime} 44^{\prime \prime} \mathrm{N}, 101^{\circ} 34^{\prime} 05^{\prime \prime} \mathrm{E}$ \\
JGC/Old Factory & Mengla & 27 & $21^{\circ} 29^{\prime} 13^{\prime \prime} \mathrm{N}, 101^{\circ} 33^{\prime} 38^{\prime \prime} \mathrm{E}$ \\
DAL/Daluo & Menghai & 47 & $21^{\circ} 42^{\prime} 37^{\prime \prime} \mathrm{N}, 100^{\circ} 12^{\prime} 23^{\prime \prime} \mathrm{E}$ \\
HHY/Huahuijiayuan & Jinghong & 32 & $22^{\circ} 00^{\prime} 21^{\prime \prime} \mathrm{N}, 100^{\circ} 47^{\prime} 03^{\prime \prime} \mathrm{E}$ \\
NKK/Nakunkang & Jinghong & 32 & $21^{\circ} 59^{\prime} 52^{\prime \prime} \mathrm{N}, 100^{\circ} 47^{\prime} 47^{\prime \prime} \mathrm{E}$ \\
GAZ/Gaozhuang & Jinghong & 32 & $22^{\circ} 00^{\prime} 33^{\prime \prime} \mathrm{N}, 100^{\circ} 49^{\prime} 13^{\prime \prime} \mathrm{E}$ \\
FTC/Fantaichang & Jinghong & 32 & $22^{\circ} 01^{\prime} 27^{\prime \prime} \mathrm{N}, 100^{\circ} 48^{\prime} 11^{\prime \prime} \mathrm{E}$ \\
ZYY/Zhouyiyuan & Jinghong & 32 & $22^{\circ} 00^{\prime} 46^{\prime \prime} \mathrm{N}, 100^{\circ} 45^{\prime} 39^{\prime \prime} \mathrm{E}$ \\
CAJ/Cangjiangzhi- & Jinghong & 32 & $22^{\circ} 00^{\prime} 25^{\prime \prime} \mathrm{N}, 100^{\circ} 45^{\prime} 24^{\prime \prime} \mathrm{E}$ \\
Zaochang & & & \\
LGD/Wushi & Guangdong 32 & $20^{\circ} 33^{\prime} 19^{\prime \prime} \mathrm{N}, 109^{\circ} 50^{\prime} 50^{\prime \prime} \mathrm{E}$ \\
LHN/Danzhou & Hainan & 32 & $19^{\circ} 31^{\prime} 16^{\prime \prime} \mathrm{N}, 109^{\circ} 34^{\prime} 51^{\prime \prime} \mathrm{E}$ \\
\hline
\end{tabular}

Abbreviation: $n$, number of mosquitoes

information are summarized in Table 2. The forward sequence of each primer was end-labelled with one of three different fluorescent dyes (FAM, ROX and HEX). Each amplification reaction was performed in a CFX96 PCR Amplification instrument in a final volume of $25 \mu \mathrm{l}(12.5 \mu \mathrm{l}$ of $2 \times$ PCR mix, each primer at $1 \mu \mathrm{M}, 1.5$ $\mu \mathrm{l}$ of DNA, and $9 \mu \mathrm{l}$ of $\mathrm{dd} \mathrm{H}_{2} \mathrm{O}$ ). The PCR conditions were as follows: $95^{\circ} \mathrm{C}$ for $10 \mathrm{~min}$, followed by 30 cycles at $95{ }^{\circ} \mathrm{C}$ for $1 \mathrm{~min}$, a different annealing temperature for each locus for $30 \mathrm{~s}$, and $72{ }^{\circ} \mathrm{C}$ for $45 \mathrm{~s}$. A final extension step was conducted at $72{ }^{\circ} \mathrm{C}$ for $10 \mathrm{~min}$. The PCR product standard mixture was analysed on the ABI3730XL Genetic Analyzer (Applied Biosystems, Foster City, USA), and the data analysed using GeneMapper software.

The genetic diversity of every locus was characterized by estimating the number of different alleles $\left(N_{a}\right)$, number of effective alleles $\left(N_{e}\right)$, Shannon's information index $(I)$, observed heterozygosity $\left(H_{o}\right)$, expected heterozygosity $\left(H_{e}\right)$ and positive inbreeding coefficient $\left(F_{I S}\right)$ with GenAIEx (version 6.501) [26]. The value of $\mathrm{Nm}$ was calculated with the formula $1 / 4\left(F_{S T}-1\right)$. PIC-Calc 0.6 [27] was used to assess polymorphic information content (PIC) across all 12 loci. Linkage disequilibrium (LD) between pairs of locations and deviations from Hardy-Weinberg equilibrium (HWE) were assessed, and analysis of molecular variance (AMOVA) was performed with Arlequin software (version 3.5.2.2) [28]. Fstat (version 2.9.3) [29] was used to compute the estimated allelic richness $(r)$ in each population. Microchecker software (version 2.2.3) [30] was used to check the presence and frequencies of null alleles at each locus.

Two approaches were used for testing genetic bottlenecks at the microsatellite loci for each population: in the first, the mean ratio of the number of alleles to the range of allele size (M ratios) was calculated to infer possible genetic bottlenecks in the more distant past [31] in Arlequin3.5 [32], and the second was performed using the infinite allele model (IAM) [33], stepwise mutation model (SMM) [34], and two-phase model (TPM) [35], which were run twice for each population, assuming that the percentage of stepwise mutations was $80 \%$. The statistical significance of the tests was assessed for each population across all loci with the Wilcoxon signed-rank test available in Bottleneck [36].

Genetic distance was calculated using GenAIEx (version 6.501) [26], and the result was used to conduct principal components analysis (PCoA) based on the codom-genotypic genetic distance. A neighbour-joining (NJ) tree was generated with Mega v.6.0 [37] based on genetic distance. Factorial correspondence analysis (FCA) was accomplished with Genetix (version 4.05) [38]. The population genetic structure was determined by a Bayesian clustering method using STRUCTURE software (version 2.3.4) [39]. A model in which allele frequencies correlated within populations was assumed ( $\lambda$ was set at 1 , the default value). The software was run with the option of admixture, allowing for some mixed ancestry within individuals, and $\alpha$ was allowed to vary. Twenty independent runs were conducted for each value of $K(K=1$ to 10$)$, with a 'burn-in' period of 50,000 iterations and 250,000 replications. The method of Evanno et al. [40] was used to determine the most likely number of clusters. The results of 20 replicate runs for each value of $K$ were combined using the Greedy algorithm of Clumpp 1.1.1 [41], and summary outputs for each value of $K$ were displayed graphically using Distruct v1.1 [42]. Isolation by distance (IBD) was estimated with Mantel's test employing the IBD Web Service using the correlation between genetic and geographic distances via regression of pairwise $F_{S T} /\left(1-F_{S T}\right)$ on the natural logarithm $(L n)$ of straight-line geographical distance [26].

\section{Mitochondrial DNA analysis}

Three mitochondrial genes, cox $1, \operatorname{nad} 4$ and $n a d 5$, were used to explore the polymorphism of all samples. The three fragments were amplified and sequenced with previously reported primers $[18,43]$. The haplotypes were numbered according to their order frequency. 
Table 2 Characteristics of the 12 microsatellite loci of Ae. aegypti used in the present study

\begin{tabular}{|c|c|c|c|c|c|}
\hline Locus & Repeat motif & Primer sequence $\left(5^{\prime}-3^{\prime}\right)$ & Annealing $T\left({ }^{\circ} \mathrm{C}\right)$ & Allele size (bp) & GenBank ID \\
\hline \multirow[t]{2}{*}{$\mathrm{AC} 10$} & $A C$ & F: CAATTATGATCCGTGGTGTT & 49 & 185 & MK733227 \\
\hline & & R: GCGAAGAATGGTGGTCTA & & & \\
\hline \multirow[t]{2}{*}{1209} & $A C$ & F: GCAATCTGGTCGTCGTTA & 54 & 370 & MK733223 \\
\hline & & R: GGCTATATCTGATCTGGTGAT & & & \\
\hline \multirow[t]{2}{*}{ AG3 } & $A G$ & F: GACTAAGCAGGACGACAG & 52 & 323 & MK733228 \\
\hline & & R: AAGCAGGTTGATGAGATTCT & & & \\
\hline \multirow[t]{2}{*}{ TTC5 } & $\mathrm{TTC}$ & F: ACATTTGTTTTGCTATTGTGG & 55 & 154 & MK733232 \\
\hline & & R: AAGAACATTATGCTAAAAAGCAG & & & \\
\hline \multirow[t]{2}{*}{ AAG6 } & AAG & F: AGGATCTTTTCGTAAGAAGCA & 55 & 150 & MK733224 \\
\hline & & R: GGAATTGTTCTCTACATGCTG & & & \\
\hline \multirow[t]{2}{*}{ AAT1 } & AAT & F: AAGGAACACTAGTTCGGTAGG & 55 & 155 & MK733225 \\
\hline & & R: GAGCTGTTCAAGAACACAAGT & & & \\
\hline \multirow[t]{2}{*}{ TTGT } & TTGT & F:TGTTTGAGCTGAAAATCTCAT & 55 & 127 & MK733233 \\
\hline & & R: GTCAAATCGGAGGTAGTGAAT & & & \\
\hline $\mathrm{AC} 1$ & $A C$ & $\begin{array}{l}\text { F: GAGTATATCGGCCTCCAATAC } \\
\text { R: CATACAGGTACACGCTAGGAT }\end{array}$ & 55 & 146 & MK733226 \\
\hline \multirow[t]{2}{*}{ CGA } & CGA & F:TGCAGTTCTACAACTCCTTTT & 55 & 156 & MK733229 \\
\hline & & R: TTACCAGTTGAAGTTGATTGC & & & \\
\hline \multirow[t]{2}{*}{ GAT } & GAT & F: CGTATCGTGTTACGCTATCTC & 55 & 154 & MK733230 \\
\hline & & R: GTAGGCAAAACGATCACAGT & & & \\
\hline \multirow[t]{2}{*}{ TC } & $\mathrm{TC}$ & F:TTCATCTTTCACTCATTCCAC & 55 & 165 & MK733231 \\
\hline & & R: CAAGTGCCCTATAGTGTTTGT & & & \\
\hline \multirow[t]{2}{*}{ AG5 } & $A G$ & F:TGATCTTGAGAAGGCATCCA & 55 & 170 & a \\
\hline & & R: CGTTATCCTTTCATCACTTGTTTG & & & \\
\hline
\end{tabular}

a Published by Slotman et al. [25]

Abbreviation: $\mathrm{T}$, temperature

Basic sequence statistics, including nucleotide diversity $(\pi)$ and the neutrality tests of Tajima and $\mathrm{Fu}$, were computed with DnaSP V5 [44]. Phylogenetic networks based on the cox 1, nad4 and nad5 sequences were constructed with the Network program (version 5.0) [45]. Similarly, IBD was estimated with Mantel's test separately for these three mitochondrial genes using the correlation between genetic and geographic distances in GenAIEx (version 6.501).

\section{Results}

\section{Quality of microsatellite markers}

A total of 585 individual mosquitoes collected from 17 populations were successfully genotyped at all 12 microsatellite loci (Additional file 1: Table S1). All loci were polymorphic, and the number of alleles ranged from 6 to 16. The average number of alleles per locus ranged from 5.98 (GAT) to 15.99 (TC). The PIC of all loci ranged from 0.199 to 0.775 , and 7 of them showed PIC values greater than 0.5 (Table 3 ). The minimum mean number of alleles of all loci was recorded for the FTC population from Jinghong (3.083) and the maximum was recorded for the CAJ population from Jinghong (6.333). The minimum $H_{o}$ was found in GEM (0.309), and the maximum $H_{o}$ was found in LYC (0.464) (Table 4). The minimum

Table 3 Polymorphic information of the 12 microsatellite loci of Ae. aegypti from Yunnan Province, China

\begin{tabular}{lllll}
\hline Locus & $\begin{array}{l}\text { No. of } \\
\text { individuals }\end{array}$ & No. of alleles & Richness & PIC \\
\hline AC10 & 585 & 15 & 14.96 & 0.722 \\
1209 & 585 & 13 & 13.00 & 0.707 \\
AG3 & 585 & 12 & 12.00 & 0.707 \\
TTC5 & 585 & 10 & 9.92 & 0.661 \\
AAG6 & 585 & 15 & 14.96 & 0.651 \\
AAT1 & 585 & 10 & 9.98 & 0.199 \\
TTGT & 585 & 15 & 15.00 & 0.775 \\
AC1 & 585 & 12 & 11.94 & 0.426 \\
CGA & 585 & 7 & 6.98 & 0.409 \\
GAT & 585 & 6 & 5.98 & 0.264 \\
TC & 585 & 16 & 15.99 & 0.631 \\
AG5 & 585 & 6 & 6.00 & 0.459 \\
Mean & & 11.42 & 11.40 & 0.551 \\
\hline
\end{tabular}


$H_{e}$ was observed in LBH (0.447), and the maximum $H_{e}$ was observed in CAJ (0.630) (Table 4). The same situation occurred for $N_{e}$ and I: the minimum value was found in NKK $\left(N_{e}=2.031, I=0.817\right)$, the maximum value was found in CAJ $\left(N_{e}=3.107, I=1.283\right)$ (Table 4).

\section{Microsatellite analysis Genetic diversity}

The highest Ho was found in the LYC population, while the lowest $H o$ was found in the GEM population, with an average value of 0.389 . The $H e$ values were higher than the $H o$ values at all sites. The HWE test suggested that all populations from Yunnan were in HWE (Additional file 2: Table S2). Similarly, 207 out of 1122 tests for linkage disequilibrium were significant after correction, while no consistency was found at any loci across all populations. The M-ratios of five populations (HHY and NKK of Jinhong, JGC of Mengla, JGL of Ruili, LYC of Longchuan) were low and indicated historical bottlenecks in these populations. The bottleneck test between the TPM and SMM models showed that there were 6 populations from Ruili and Jinghong (MML, RJL and JGL of Ruili, FTC, ZYY and CAJ of Jinhong) that had experienced recent population extinction (Table 4).

\section{Genetic structure}

Bayesian clustering analysis with STRUCTURE in these populations identified the most likely number of clusters as 3: the samples from Ruili, Longchuan and Gengma were in the first cluster (Group 1); the samples from Jinghong and Menghai made up the second cluster (Group 2); and the populations from Mengla, Guangdong and Hainan belonged to the third group, which showed a close relationship among these populations (Group 3) (Fig. 2a). The map of PCoA (Fig. 2b) and the UPGMA tree (Fig. 3) showed similar results, indicating 3 groups, as indicated above.

\section{Genetic differences}

The $F_{I S}$ values based on 12 loci were positive across all populations (Additional file 2: Table S3), which indicated different degrees of inbreeding within populations. The maximum $F_{I S}$ value was found in GEM (0.486), and the minimum $F_{I S}$ value was found in FTC (0.071). The $F_{S T}$ and $N m$ values are shown in Additional file 2: Table S4. The lowest pairwise $F_{S T}$ value among the 17 populations was found between MML and NAM $\left(F_{S T}=0.017\right)$, and these two populations were both from Ruili city. The pairwise $F_{S T}$ values were always lower between populations from the same city than between those from different cities. The $F_{S T}$ values between LGD, LHN and other

Table 4 Genetic variation in 17 populations of Ae. aegypti from Yunnan Province, China, averaged over 12 microsatellite loci

\begin{tabular}{|c|c|c|c|c|c|c|c|c|c|c|c|}
\hline Pop & $N$ & $N_{a}$ & $N_{e}$ & 1 & $H_{0}$ & $H_{e}$ & $u H_{e}$ & $F_{1 S}$ & IAM & TPM & SMM \\
\hline MML & 46.583 & 5.583 & 2.505 & 1.029 & 0.407 & 0.519 & 0.525 & 0.280 & 0.564 & 0.419 & 0.003 \\
\hline NAM & 35.000 & 4.167 & 2.507 & 0.957 & 0.421 & 0.507 & 0.514 & 0.165 & 0.113 & 0.117 & 0.102 \\
\hline RJL & 32.000 & 4.333 & 2.366 & 0.929 & 0.378 & 0.492 & 0.500 & 0.245 & 0.280 & 0.478 & 0.026 \\
\hline$J G L$ & 46.750 & 4.667 & 2.182 & 0.856 & 0.417 & 0.455 & 0.460 & 0.140 & 0.390 & 0.585 & 0.008 \\
\hline LYC & 31.833 & 4.917 & 2.623 & 1.051 & 0.464 & 0.547 & 0.556 & 0.137 & 0.337 & 0.595 & 0.185 \\
\hline $\mathrm{LBH}$ & 27.917 & 3.750 & 2.237 & 0.834 & 0.353 & 0.447 & 0.455 & 0.145 & 0.363 & 0.367 & 0.318 \\
\hline$J G C$ & 27.000 & 3.833 & 2.412 & 0.852 & 0.346 & 0.452 & 0.461 & 0.217 & 0.175 & 0.184 & 0.305 \\
\hline $\mathrm{DAL}$ & 46.500 & 4.750 & 2.612 & 1.033 & 0.428 & 0.550 & 0.556 & 0.197 & 0.146 & 0.350 & 0.197 \\
\hline GEM & 22.250 & 5.250 & 2.854 & 1.135 & 0.309 & 0.580 & 0.594 & 0.484 & 0.169 & 0.179 & 0.059 \\
\hline $\mathrm{HHY}$ & 31.917 & 3.333 & 2.324 & 0.843 & 0.434 & 0.477 & 0.485 & 0.103 & 0.045 & 0.056 & 0.470 \\
\hline NKK & 32.000 & 3.750 & 2.031 & 0.817 & 0.378 & 0.470 & 0.478 & 0.231 & 0.472 & 0.501 & 0.210 \\
\hline GAZ & 31.500 & 3.833 & 2.288 & 0.922 & 0.422 & 0.519 & 0.528 & 0.254 & 0.249 & 0.266 & 0.432 \\
\hline FTC & 31.583 & 3.083 & 2.170 & 0.796 & 0.436 & 0.461 & 0.469 & 0.039 & 0.007 & 0.047 & 0.443 \\
\hline ZYY & 31.917 & 3.417 & 2.304 & 0.863 & 0.393 & 0.489 & 0.496 & 0.153 & 0.007 & 0.049 & 0.423 \\
\hline CAJ & 31.250 & 6.333 & 3.107 & 1.283 & 0.335 & 0.630 & 0.640 & 0.456 & 0.610 & 0.385 & 0.003 \\
\hline LGD & 31.667 & 3.500 & 2.180 & 0.829 & 0.328 & 0.457 & 0.464 & 0.300 & & & \\
\hline LHN & 31.500 & 3.417 & 2.271 & 0.809 & 0.361 & 0.448 & 0.455 & 0.167 & & & \\
\hline Mean & 33.480 & 4.230 & 2.410 & 0.932 & 0.389 & 0.500 & 0.508 & 0.222 & & & \\
\hline
\end{tabular}

Notes: $P$-values for genetic bottleneck detection using the Wilcoxon signed-rank test under the infinite allele (IAM), step-wise mutation (SMM) and two-phase mutation (TPM) models. Bold characters denote a significant heterozygote deficiency $(P<0.05)$ after correction for multiple testing by the sequential Bonferroni procedure

Abbreviations: $N$, effective number of samples; $N_{a}$, number of alleles per population; $N_{e^{\prime}}$ effective allele; $I$, Shannon's information index; $H_{o}$, observed heterozygosity; $H_{e}$, expected heterozygosity; $u H_{e}$, unbiased expected heterozygosity; $F_{I S}$, inbreeding coefficient 


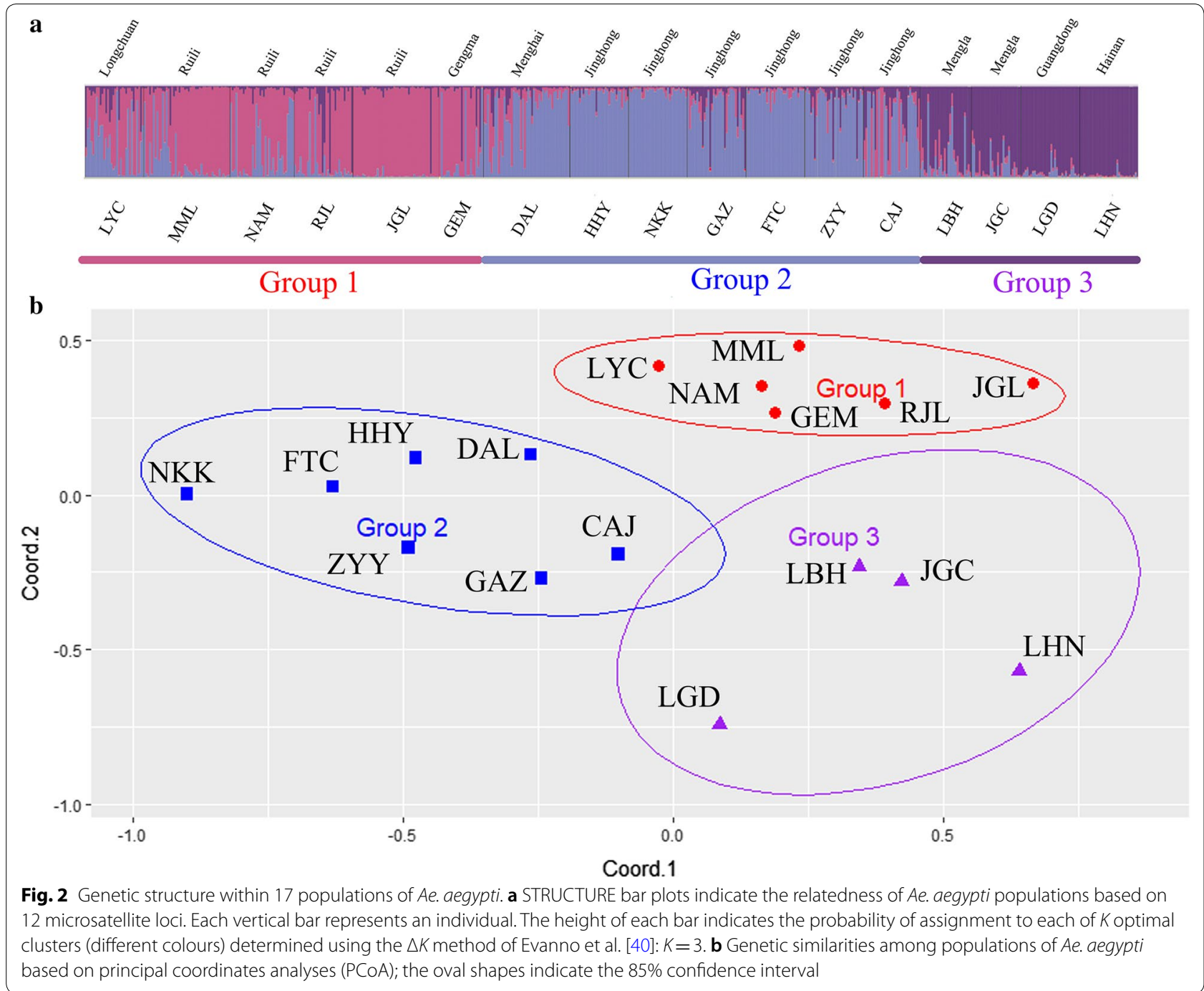

populations were especially high compared to those for other populations from Yunnan. The value of $N_{m}$ was between 0.807 (LHN and NKK) and 14.339 (MML and NAM), and most of the $N_{m}$ values among the populations were greater than 1 , indicating that there was a certain degree of communication among these populations of Ae. aegypti. According to the AMOVA results, the percentage of variation between groups was only $8.71 \%$, while the percentage of variation within individuals was $63.48 \%$, and the $F_{I T}$ (total inbreeding coefficient) value was 0.3652 . The results of the Mantel test showed a significant positive correlation between Nei's standard genetic distance and geographical distance $\left(R^{2}=0.3747\right.$, $P<0.01)$, as shown in Fig. 4a.

\section{DNA sequencing analysis}

There were 549 mtDNA cox 1 (507 bp), 544 mtDNA nad4 (553 bp) and 534 mtDNA nad5 (414 bp) nucleotide sequences obtained from the 17 populations.

The mitochondrial DNA results showed that the cox 1 gene had 11 haplotypes. The haplotype diversity index of the total population was 0.5670 , and the nucleotide diversity index was 0.0034 . The average number of nucleotide differences $(k)$ was 1.687. The LYC population harboured the most haplotypes $(n=4)$ : Hap1, Hap9, Hap10 and Hap11. There were 5 populations (LGD, LHN, MML, NAM and RJL) exhibiting only one type of haplotype. The nad4 gene had 15 haplotypes. The haplotype diversity index of the total population was 0.5603 , and the nucleotide diversity index was 0.0224 . The MML population from Ruili and the DAL population from Menghai exhibited the most 




Fig. 3 UPGMA cluster analysis based on the genetic distance of all populations, showing the relationships between sampled cities. The red, blue and purple indicate Group 1, Group 2 and Group 3, respectively

haplotypes $(n=5)$. Four of the 5 populations with only one haplotype of nad4 were the same as those with only one cox 1 haplotype; these populations were LGD, LHN, NAM, RJL and HHY. The nad5 gene presented 10 haplotypes, and Hap1 was detected in all geographical populations. The indices of the total population were 0.503 for haplotype diversity, 0.005 for nucleotide diversity and 1.920 for the average number of nucleotide differences.

The results of the population expansion test showed that the $P$-values with negative test values were all less than 0.05 , indicating that these populations had not expanded during their history. The haplotype network maps (Fig. 5) showed that each mitochondrial gene exhibited two dominant haplotypes. One was found in a large number of individuals from Jinghong and Menghai, and the other was found in many individuals collected in Ruili, Longchuan and Gengma. Most of the other haplotypes differed from these two haplotypes. No correlation was detected between genetic and geographic distances for all three mtDNA genes $\left(\operatorname{cox} 1: R^{2}=0.011, P<0.05\right.$; nad4: $R^{2}=0.019, P<0.140:$ nad5: $\left.R^{2}=0.031, P<0.870\right)$, as shown in Fig. 4b-d.

\section{Discussion}

Aedes aegypti is a mosquito species distributed worldwide in tropical and subtropical regions. No Ae. aegypti were found in any survey conducted before 2000 in Yunnan, and $A e$ aegypti larvae were captured for the first time at the Jiegao Port of Ruili in 2002. The larvae of Ae. aegypti are mainly found in jars and flowerpots indoors and outdoors. Female mosquitoes usually suck blood near their breeding grounds. The flying distance of adults is usually short, and the average diffusion radius is within $100 \mathrm{~m}$ [46]. Aedes aegypti does not enter diapause in low temperatures and short photoperiods [47] and therefore cannot expand over long distances via diapause eggs, which is the main reason that Ae. albopictus expanded from Asia to North America and Europe [48, 49]. The mode of Ae. aegypti expansion in border areas of China and Southeast Asian countries could be revealed via population genetics research with microsatellite and mtDNA markers.

\section{Genetic diversity and demographic history}

Genetic diversity is one of the most important attributes of populations. The decline in genetic diversity among geographical populations could be an indicator of the bottleneck effect or founder effect that occurred in the history of populations expanding or invasion [50]. 

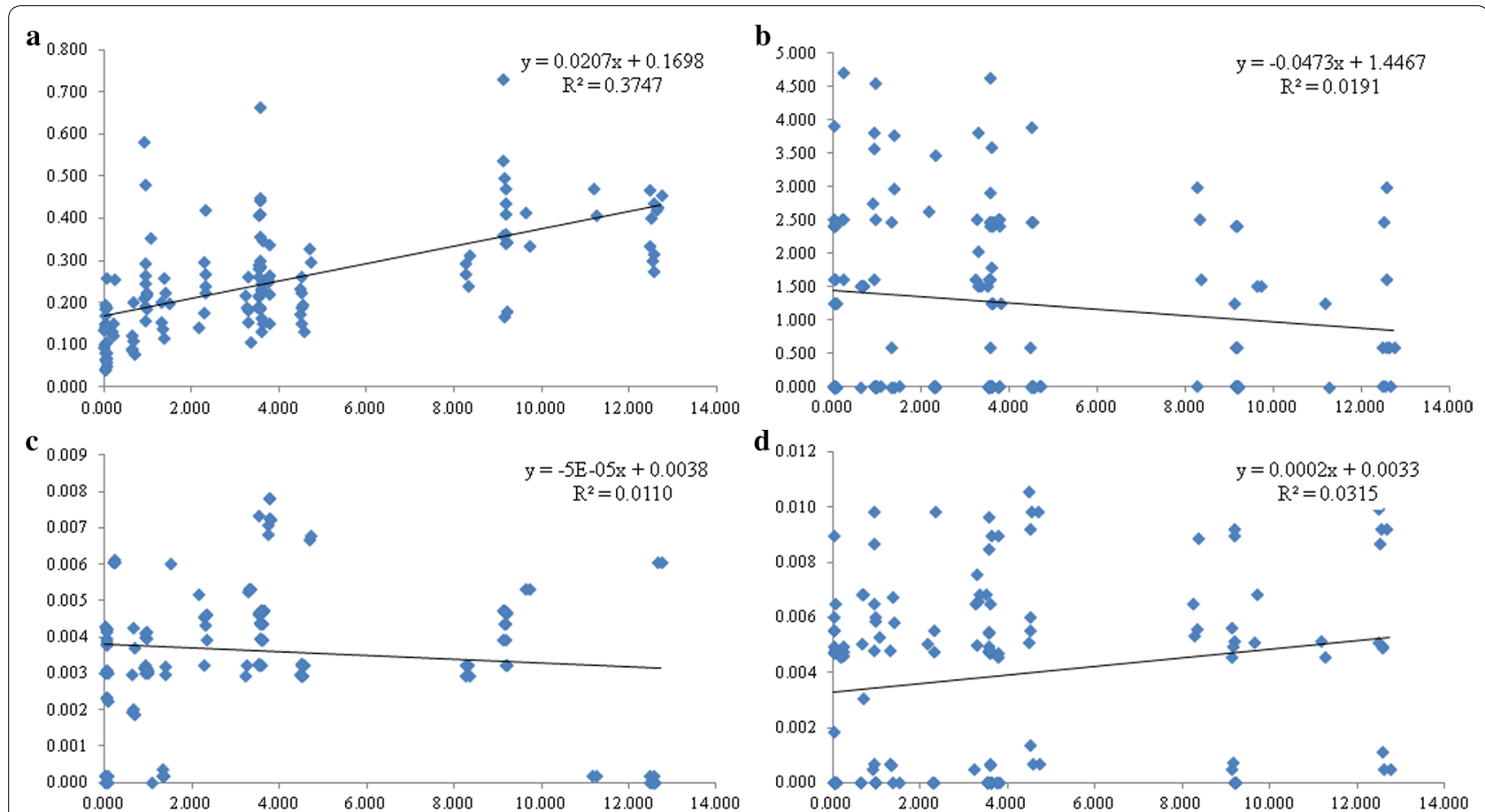

Fig. 4 Analysis of the relationship between genetic distance $\left[F_{S T} /\left(1-F_{S T}\right)\right]$ and geographical distance $[\mathrm{ln}(\mathrm{km})]$ based on 12 microsatellite loci $\left(R^{2}=0.3747, P<0.01\right)(\mathbf{a}), \operatorname{cox} 1\left(R^{2}=0.011, P<0.05\right)(\mathbf{b}), \operatorname{nad4}\left(R^{2}=0.0191, P<0.140\right)(\mathbf{c})$, and $\operatorname{nad5}\left(R^{2}=0.0315, P<0.870\right)(\mathbf{d})$

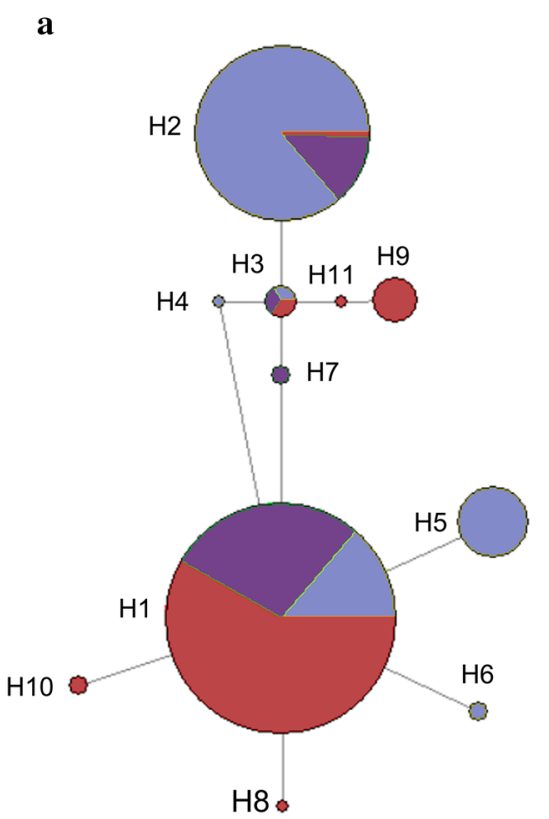

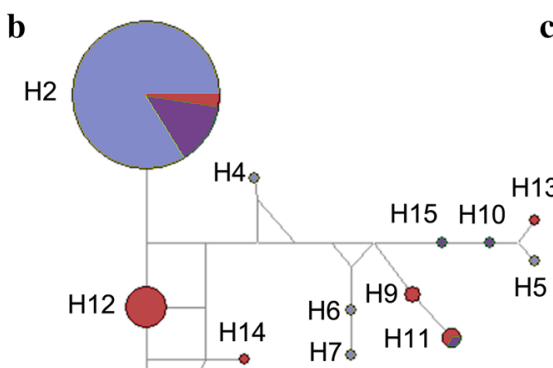



Fig. 5 Genealogical relationships of Ae. aegypti based on the median-joining haplotype network of 3 mitochondrial fragments: cox 1 (a); nad4 (b) and nad5 (c). Each line in the network represents a single nucleotide mutation. The size of the nodes corresponds to the frequency of the haplotypes. Colours indicate the collection regions: Group 1 (red), Group 2 (blue), and Group 3 (purple) 
According to the results of this study, the genetic diversities of the different populations are similar, with $H_{o}$ values ranging from 0.309 to 0.467 , with an average value of 0.389 . All the natural populations conformed to HardyWeinberg equilibrium. These results indicated that there was no obvious decline in genetic diversity among these populations, which indicated that the Ae. aegypti populations from these areas were not the offspring of one or two invasion events from Southeast Asian countries but the result of successive expansions via different routes.

The results of the bottleneck tests (Table 4) showed two scenarios of the demographic history of Ae aegypti of Yunnan. The M-ratio test indicated that five populations had experienced slight bottlenecks in their history because the $\mathrm{M}$-value of these populations was just below the critical value of 0.68 proposed by Garza \& Williamson [31] and that the other populations had not suffered bottlenecks in their distant past. The results of Wilcoxon signed-rank tests showed that only some populations from Ruili and Jinghong had experienced bottlenecks recently. The first scenario meant that Ae. aegypti expanded naturally from Southeast Asia to Yunnan, rather than being imported with human activities, and the second indicated that only some Ae. aegypti populations in urban areas have been under pressure from mosquito control measures applied during dengue fever outbreaks in recent years.

\section{Genetic structure and differentiation}

The results of population clustering were consistent with the geographical distribution of the populations. Longchuan, Ruili and Gengma are located in the southwestern part of Yunnan, contiguous with Myanmar. Populations from these areas were clustered in group 1 (Figs. 2, 3). The second group was composed of populations from Jinghong and Menghai, which are contiguous with northeastern Myanmar. The third group included two populations from Mengla, which is the only county of Xishuangbanna bordering Laos. This result suggests that the populations from these areas exhibited at least three sources and that expansion had occurred independently from adjacent areas of Myanmar and Laos. According to the results of genetic structure and PCoA, two laboratory populations (LBG and LBH) were closely related to populations from Mengla. Among the six counties, only Mengla is adjacent to Laos, which could be the reason that populations from Mengla are separating from other populations. Because Laos is economically closely related to Vietnam, and Vietnam is geographically close to Hainan and Guangdong, the populations of Ae. aegypti from Guangdong and Hainan are relatively closely related to populations of Mengla.

The results of the IBD test based on microsatellite markers showed that there was a significant positive correlation between geographical distance and genetic distance $\left(R^{2}=0.375\right)$ (Fig. 4a). This finding suggested that the expansion of Ae. aegypti in collection areas was a natural process, rather than a process caused by human activities. However, the IBD tests based on mtDNA markers did not show a positive correlation, which suggested that the mutation rate of the mtDNA markers was lower than that of microsatellite markers and that the expansion of Ae. aegypti in these areas was a recent event.

\section{The cause of Ae. aegypti expansion in southwestern Yunnan}

Because of the close relationship with dengue fever, considerable attention has been paid to the occurrence of $A e$. aegypti in Yunnan by public health authorities and academic institutes. Local CDC entities carry out specific surveillance in border areas of Yunnan [7]. Shi et al. [51] studied the population genetics of Ae. aegypti populations collected from six counties of Yunnan. Their results showed that all populations could be divided into three groups, two of which included populations from Jinghong and Ruili, respectively, and the third included populations from the other four counties (Menghai, Mengla, Gengma and Longchuan), which was different from the results of the present study. This relationship was not in accordance with the geographical relationship of populations, and could not be explained with any reasonable scenario of the expansion of Ae. aegypti in this area.

The occurrence of Ae. aegypti in high-latitude areas has not only been reported in southern Yunnan, China. Positive breeding sites of Ae. aegypti were noted at Darjeeling, West Bengal, India in 2006 [52]. The synchronous invasion of Ae. aegypti in northern South and Southeast Asia suggested that the northward expansion of this species was related to climate change on a large spatial scale. From 1954 to 2006, the annual mean temperature of Jinghong increased by approximately $1.3{ }^{\circ} \mathrm{C}$, from $21.8{ }^{\circ} \mathrm{C}$ to $23.1{ }^{\circ} \mathrm{C}$, and the increase was more obvious from the 1980s onward [53]. Therefore, the warmer climate of southwestern Yunnan should be the primary factor responsible for the expansion of Ae. aegypti in these areas. However, because this species does not enter diapause, its occurrence in other areas of Yunnan will be determined by the critical low temperature threshold for the survival of this species [54].

Hlaing et al. [55] studied the population genetics of $A e$. aegypti populations from mainland Southeast Asia. The results suggested that human transportation routes have resulted in passive long-distance migration of Ae. aegypti in these areas $\left(R^{2}=0.111, P<0.05\right)$. However, the results of the IBD test in the present study $\left(R^{2}=0.375, P<0.01\right)$ indicated the role of human transportation in the expansion of Ae. aegypti in southwestern Yunnan was not as 
significant as in Southeast Asia. Aedes aegypti occurred independently in different areas in southwestern Yunnan from 2003 onward, indicating only a short history of these invasions. Because of the complex topography of Yunnan, transportation among these areas (Ruili, Gengma and Jinghong) is not convenient. Thus, the expansion of Ae. aegypti among these areas has not been found according to the results of the present study, and the distribution areas of the species were still restricted to border areas as of 2017 [56].

\section{Implications for vector surveillance}

After the invasion of Ae. aegypti in Yunnan, dengue fever outbreaks were reported from four county areas of Yunnan (Ruili, Gengma, Jinghong and Mengla) [56]. Dengue fever became the most important vector-borne disease in Yunnan in 2013 [57]. Since the first report of Ae. aegypti in Yunnan, this species has only been found in border areas, although it was reported in eight county areas in 2017 [56]. There has been no evidence of expansion of distribution areas from border areas to inland areas of Yunnan. According to the results of the present study, Ae. aegypti invaded different areas of Yunnan independently, and there is no evidence of expansion among these areas. Therefore, the most important measures that can be adopted by the public health authority of Yunnan are surveillance and control of Ae. aegypti in border areas, especially in counties in areas that are currently negative for Ae. aegypti.

\section{Conclusions}

The populations of Ae. aegypti in Yunnan can be divided into three groups according to population genetics. The genetic relationships of these populations were concurrent with their geographical relationships. This result suggested that the occurrence of Ae. aegypti in Yunnan is a result of the expansion of its distribution areas, rather than imported events closely related to human activity. Thus far, populations of Ae. aegypti have only occurred in border areas of Yunnan, and the species has not colonized inland counties of Yunnan. According to the topographic and climatic differences between border areas and inland areas of Yunnan, it should be difficult for the population of Ae. aegypti to expand to inland areas of Yunnan in a short time. Therefore, efforts aimed at the surveillance of Ae. aegypti and control of dengue fever should focus on the border areas of Yunnan, especially the counties and ports that are currently free of Ae. aegypti.

\section{Supplementary information}

Supplementary information accompanies this paper at https://doi. org/10.1186/s13071-019-3818-8.
Additional file 1: Table S1. The microsatellite short tandem repeat data.

Additional file 2: Table S2. Results of Hardy-Weinberg equilibrium. Table S3. $F_{\text {IS }}$ per population. Table S4. Population $F_{S T}$ (lower left) and $N_{m}$ (top right) matrix table.

\begin{abstract}
Abbreviations
AMOVA: analysis of molecular variance; cox1: cytochrome c oxidase subunit 1 gene; DF: dengue fever; FCA: factorial correspondence analysis; HWE: Hardy-Weinberg equilibrium; IAM: infinite allele model; IBD: isolation by distance; LD: linkage disequilibrium; mtDNA: mitochondrial DNA; nad4: NADH dehydrogenase subunit 4 gene; nad5: NADH dehydrogenase subunit 5 gene; $\mathrm{NJ}$ : neighbour-joining; PCoA: principal components analysis; PIC: polymorphic information content; SMM: stepwise mutation model; TPM: two-phase model; WHO: World Health Organization.
\end{abstract}

\section{Acknowledgements}

We thank Jianhong Zou of the Jinghong CDC, Yonghua Liu and Xiaoxiong Yin of the Ruili CDC, and Huaxing Liu and Jiangyun Liu of the Mengla CDC for their help in sample collection. The manuscript has been improved by comments from the editor and anonymous reviewers.

\section{Authors' contributions}

PL took part in fieldwork, performed molecular analyses and interpreted the data, and drafted the manuscript. YG, JJ and DY undertook field sampling for this research. LL designed the research, took part in field work, polished the manuscript and provided financial support for the research. QL designed the research study and provided the financial support. All authors discussed the results and commented on the manuscript. All authors read and approved the final manuscript.

\section{Funding}

This work was supported by the Ministry of Science and Technology of the People's Republic of China National Important Scientific \& Technology Project (2018ZX10101002-002 to QL), and the National Key R\&D Programme of China (2016YFC1200503 to LL)

\section{Availability of data and materials}

Data supporting the conclusions of this article are included within the article and its additional files. The microsatellite short tandem repeat dataset generated during this study are provided in Additional file 1: Table S1. The newly generated mitochondrial DNA sequences were submitted to the GenBank database under the accession numbers MK984840-MK985388 (cox1 gene); MK985923-MK986466 (nad4 gene); MK985389-MK985922 (nad5 gene).

Ethics approval and consent to participate

Not applicable.

\section{Consent for publication}

Not applicable.

\section{Competing interests}

The authors declare that they have no competing interests.

\section{Author details}

${ }^{1}$ State Key Laboratory of Infectious Disease Prevention and Control, National Institute for Communicable Disease Control and Prevention, Chinese Center for Disease Control and Prevention, Beijing 102206, China. ${ }^{2}$ Yunnan Institute of Parasitic Diseases, Pu'er 665000, China.

Received: 11 July 2019 Accepted: 20 November 2019

Published online: 27 November 2019

References

1. Tatem AJ, Hay SI, Rogers DJ. Global traffic and disease vector dispersal. Proc Natl Acad Sci USA. 2006;103:6242. 
2. World Health Organization. Special programme for research, training in tropical diseases. Dengue: guidelines for diagnosis, treatment, prevention and control. Geneva: World Health Organization; 2009.

3. Bhatt $\mathrm{S}$, Gething PW, Brady OJ, Messina JP, Farlow AW, Moyes $\mathrm{CL}$, et al. The global distribution and burden of dengue. Nature. 2013;496:504-7.

4. WHO. Dengue control/What is dengue? Geneva: World Health Organization; 2019. https://www.who.int/denguecontrol/disease/en/. Accessed 15 Sept 2019

5. Lu B. Fauna Sinica, Insecta Vol. 9, Diptera: Culicidae II, vol. 8. Beijing: Science Press; 1997.

6. Dong X, Cai F, Zhou H, Wang X, Dong L, Wu C, et al. Mosquitoes investigation in the border ports of Yunnan. Chin J Vector Biol Control. 2004;15:142-5.

7. Yang $\mathrm{M}$, Jiang J, Zheng $\mathrm{Y}$, Zhou $\mathrm{H}$. Distribution survey on Aedes aegypti in the border areas of Yunnan Province, China. Chin J Vector Biol Control. 2015;26:406-8

8. Zhao Y, Li L, Ma D, Jia L, Ma Z, Wang X, et al. Molecular characterization and viral origin of the 2015 dengue outbreak in Xishuangbanna, Yunnan, China. Sci Rep. 2016;6:34444

9. Sang S, Wang S, Lu L, Bi P, Lv M, Liu Q. The epidemiological characteristics and dynamic transmission of dengue in China, 2013. PLos Negl Trop Dis. 2016;10:e0005095.

10. Urdaneta-Marquez L, Failloux AB. Population genetic structure of Aedes aegypti, the principal vector of dengue viruses. Infect Genet Evol. 2011;11:253.

11. Sang $S$, Chen $B, W u H$, Yang Z, Di B, Wang $L$, et al. Dengue is still an imported disease in China: a case study in Guangzhou. Infect Genet Evol. 2015;32:178-90

12. Balloux F, Lugon-Moulin N. The estimation of population differentiation with microsatellite markers. Mol Ecol. 2010;11:155-65.

13. Swaegers J, Mergeay J, Maes GE, Houdt JKJV, Larmuseau MHD, Stoks R. Microsatellite marker development and putative SNP detection for a northward expanding damselfly species using next generation sequencing. Conserv Genet Resour. 2012;4:1079-84.

14. Chambers EW, Meece JK, Mcgowan JA, Lovin DD, Hemme RR, Chadee $\mathrm{DD}$, et al. Microsatellite isolation and linkage group identification in the yellow fever mosquito Aedes aegypti. J Hered. 2007;98:202-10.

15. Vidal PO, Suesdek L. Comparison of wing geometry data and genetic data for assessing the population structure of Aedes aegypti. Infect Genet Evol. 2012;12:591-6.

16. Lovin DD, Washington KO, Bruyn BD, Hemme RR, Mori A, Epstein SR, et al. Genome-based polymorphic microsatellite development and validation in the mosquito Aedes aegypti and application to population genetics in Haiti. BMC Genomics. 2009;10:590.

17. Gloria-Soria A, Ayala D, Bheecarry A, Calderon-Arguedas O, Chadee DD, Chiappero M, et al. Global genetic diversity of Aedes aegypti. Mol Ecol. 2016:25:5377-95.

18. Mousson L, Dauga C, Garrigues T, Schaffner F, Vazeille M, Failloux AB. Phylogeography of Aedes (Stegomyia) aegypti (L.) and Aedes (Stegomyia) albopictus (Skuse) (Diptera: Culicidae) based on mitochondrial DNA variations. Genet Res. 2005:86:1-11.

19. Behura SK, Lobo NF, Haas B, Debruyn B, Lovin DD, Shumway MF, et al. Complete sequences of mitochondrial genomes of Aedes aegypti and Culex quinquefasciatus and comparative analysis of mitochondrial DNA fragments inserted in the nuclear genomes. Insect Biochem Mol. 2011:41:770-7.

20. Beard C, Hamm D, Collins F. The mitochondrial genome of the mosquito Anopheles gambiae: DNA sequence, genome organization, and comparisons with mitochondrial sequences of other insects. Insect Mol Biol. 2010:2:103-24.

21. Chan A, Chiang LP, Hapuarachchi HC, Tan CH, Pang SC, Lee R, et al. DNA barcoding: complementing morphological identification of mosquito species in Singapore. Parasit Vectors. 2014;7:569.

22. Gorrochotegui-Escalante N, Munoz M, Fernandez-Salas I, Beaty B. Genetic isolation by distance among Aedes aegypti populations along the northeastern coast of Mexico. Am J Trop Med Hyg. 2000:62:200-9.

23. Gorrochotegui-Escalante N, Gomez-Machorro C, Lozano-Fuentes S, Fernandez-Salas L, De Lourdes Munoz M, Farfan-Ale JA, et al. Breeding structure of Aedes aegypti populations in Mexico varies by region. Am J Trop Med Hyg. 2002;66:213-22.
24. De Merida A Palmieri M, Yurrita M, Molina A, Molina E Mitochondrial DNA variation among Anopheles albimanus populations. Am J Trop Med Hyg. 1999;61:230-9.

25. Slotman MA, Kelly NB, Harrington LC, Kitthawee S, Jones JW, Scott TW, et al. Polymorphic microsatellite markers for studies of Aedes aegypti (Diptera: Culicidae), the vector of dengue and yellow fever. Mol Ecol Notes. 2010;7:168-71.

26. Peakall R, Smouse PE. GenAIEx 6.5: genetic analysis in Excel. Population genetic software for teaching and research—an update. Bioinformatics. 2012;28:2537-9.

27. Nagy S, Poczai P, Cernák I, Gorji AM, Hegedűs G, Taller J. PICcalc: an online program to calculate polymorphic information content for molecular genetic studies. Biochem Genet. 2012;50:670-2.

28. Laurent E, Guillaume L, Stefan S. Arlequin (version 3.0): an integrated software package for population genetics data analysis. Evol Bioinform. 2005;1:47-50.

29. Goudet J. FSTAT, a program to estimate and test gene diversities and fixation indices. Lausanne: Université de Lausanne; 2005. https://www2.unil. ch/popgen/softwares/fstat.htm. Accessed 15 Sept 2019.

30. Van Oosterhout C, Hutchinson WF, Wills DPM, Shipley P. MICRO-CHECKER: software for identifying and correcting genotyping errors in microsatellite data. Mol Ecol Resour. 2010;4:535-8.

31. Garza JC, Williamson EG. Detection of reduction in population size using data from microsatellite loci. Mol Ecol. 2001;10:305-18.

32. Excoffier L, Lischer H. Arlequin suite ver 3.5: a new series of programs to perform population genetics analyses under Linux and Windows. Mol Ecol Resour. 2010;10:564-7.

33. Crow JF, Kimura M. An introduction to population genetics. New York: Harper \& Row; 1970.

34. Ohta T, Kimura M. A model of mutation appropriate to estimate the number of electrophoretically detectable alleles in a finite population. Genet Res. 1973;22:201

35. Di Rienzo A, Peterson AC, Garza JC, Valdes AM, Slatkin M, Freimer NB. Mutational processes of simple-sequence repeat loci in human populations. Proc Natl Acad Sci USA. 1994:91:3166.

36. Peery MZ, Kirby R, Reid BN, Stoelting R, Doucet-Bëer E, Robinson S, et al. Reliability of genetic bottleneck tests for detecting recent population declines. Mol Ecol. 2012;21:3403-18.

37. Tamura K, Stecher G, Peterson D, Filipski A, Kumar S. MEGA6: molecular evolutionary genetics analysis version 6.0. Mol Biol Evol. 2013;2725:9.

38. Belkhir K, Borsa P, Chikhi L, Raufaste N, Bonhomme F. GENETIX 4.05, logiciel sous Windows TM pour la génétique des populations Laboratoire Génome, Populations, Interactions, CNRS UMR 5171, Université de Montpellier II, 1996. Montpellier: Université de Montpellier II; 2004.

39. Pritchard JK, Stephens M, Donnelly P. Inference of population structure using multilocus genotype data. Genetics. 2000;155:945-59.

40. Evanno G, Regnaut S, Goudet J. Detecting the number of clusters of individuals using the software STRUCTURE: a simulation study. Mol Ecol. 2005:14:2611-20.

41. Jakobsson M, Rosenberg NA. CLUMPP: a cluster matching and permutation program for dealing with label switching and multimodality in analysis of population structure. Bioinformatics. 2007;23:1801.

42. Rosenberg NA. DISTRUCT: a program for the graphical display of population structure. Mol Ecol Resour. 2010;4:137-8.

43. Bracco JE, Capurro ML, Lourençodeoliveira R, Sallum MA. Genetic variability of Aedes aegypti in the Americas using a mitochondrial gene: evidence of multiple introductions. Mem Inst Oswaldo Cruz. 2007;102:573-80.

44. Librado P, Rozas J. DnaSP v5: a software for comprehensive analysis of DNA polymorphism data. Bioinformatics. 2009;25:1451-2.

45. Bandelt HJ, Forster P, Röhl A. Median-joining networks for inferring intraspecific phylogenies. Mol Biol Evol. 1999;16:37-48.

46. Reiter P, Amador MA, Anderson RA, Clark GG. Dispersal of Aedes aegypti in an urban area after blood feeding as demonstrated by rubidium-marked eggs. Am J Trop Med Hyg. 1995;52:177-9.

47. Christophers R. Aedes aegypti (L.) The yellow fever mosquito. Its life history, bionomics and structrue. New York-London: Cambridge University Press; 1960.

48. Hawley WA, Reiter P, Copeland RS, Pumpuni CB, Craig GB. Aedes albopictus in North America: probable introduction in used tires from northern Asia. Science. 1987:236:1114-6. 
49. Roche B, Léger L, L'Ambert G, Lacour G, Foussadier R, Besnard G, et al. The spread of Aedes albopictus in metropolitan France: contribution of environmental drivers and human activities and predictions for a near future. PLOS ONE. 2015;10:e0125600.

50. Begon M, Townsend CR, Harper JL. Ecology: from individuals to ecosystems. 4th ed. Oxford: Blackwell; 2006.

51. Shi QM, Zhang HD, Wang G, Guo XX, Xing D, Dong YD, et al. The genetic diversity and population structure of domestic Aedes aegypti (Diptera: Culicidae) in Yunnan Province, southwestern China. Parasit Vectors. 2017;10:292.

52. Gautam A, Pramanik MK, Saha GK. Immatures of Aedes aegypti in Darjeeling Himalayas-expanding geographical limits in India. Indian J Med Res. 2009;129:455-7.

53. Cheng J, Xie M. The analysis of regional climate change features over Yunnan in recent 50 years. Prog Geogr. 2008;27:19-26.
54. Tsai PJ, Lin TH, Teng HJ, Yeh HC. Critical low temperature for the survival of Aedes aegypti in Taiwan. Parasit Vectors. 2018;11:22.

55. Hlaing T, Tun-Lin W, Somboon P, Socheat D, Setha T, Min S, et al. Spatial genetic structure of Aedes aegypti mosquitoes in mainland Southeast Asia. Evol Appl. 2010;3:319-39.

56. Li C, Dong X, Yang M. Geographical distribution and seasonal variations of Aedes aegypti in Yunnan Province. Chin J Vector Biol Control. 2018:29:394-6.

57. Lai S, Huang Z, Hang Z, Anders KL, Perkins TA, Yin W, et al. The changing epidemiology of dengue in China, 1990-2014: a descriptive analysis of 25 years of nationwide surveillance data. BMC Med. 2015;13:100.

\section{Publisher's Note}

Springer Nature remains neutral with regard to jurisdictional claims in published maps and institutional affiliations.
Ready to submit your research? Choose BMC and benefit from:

- fast, convenient online submission

- thorough peer review by experienced researchers in your field

- rapid publication on acceptance

- support for research data, including large and complex data types

- gold Open Access which fosters wider collaboration and increased citations

- maximum visibility for your research: over $100 \mathrm{M}$ website views per year

At BMC, research is always in progress.

Learn more biomedcentral.com/submissions 